$\mathrm{H}$

Von Thomas Korbun

und Alexandra Dehnhardt Konjunktur: irre Kühe, Schweine vollgepumpt mit Antibiotika, Klärschlamm und Tierreste im Futtertrog. Angeekelt fasziniert blicken wir auf eine nicht enden wollende Kette schier unglaublicher Enthüllungen - die am Ende doch nur Altbekanntes offenbart: die industrialisierte Landwirtschaft in ihrer heutigen Form zerstört die natürlichen Lebensgrundlagen. Und dies spiegelt sich nicht zuletzt auch in abnehmender Qualität und Sicherheit ihrer Produkte wider. Was mit zunehmender Technisierung, mit erhöhtem Einsatz von Kunstdüngern und Pestiziden begann, dieser ungebrochene Trend serviert uns heute BSE, Hormone und fades Gemüse zum Mittagessen.

Die aktuelle Krise wird zu Recht als GAU der konventionellen Land- und Ernährungswirtschaft gesehen. Alleine darauf aber eine Argumentation für eine Wende in der Agrarpolitik aufzubauen, könnte sich spätestens dann als fataler Fehler erweisen, wenn das erste BioRind mit BSE auftaucht. Und damit ist zu rechnen. Damit die öffentliche Diskussion dann nicht unvermittelt implodiert, muss sie neben den aktuellen Skandalen auch ,,alte“ Argumente wieder auf die politische Agenda zu setzen: etwa die Belastung von Grundwasser und Oberflächengewässern mit Nährstoffen und Pestiziden, degradierende Bodennutzung, Artenverluste oder die schleichende Zerstörung einmaliger Kulturlandschaften.

Die Zeit scheint günstiger als je zuvor, der alten Agrarpolitik den Rücken zuzukehren, die Wende $\mathrm{zu}$ einer nachhaltigeren Landwirtschaft ist in greifbare Nähe gerückt. Die Verbraucher sind alarmiert, die Agrarlobby von der Skandalwelle geschwächt und die Politik endlich bereit, überkommene Positionen aufzubrechen. Das noch geöffnete Zeitfenster sollte die Politik entschlossen für zentrale Weichenstellungen nutzen.

Was ist zu tun? Die Dynamik trifft die jetzt handelnden Politiker unvorbereitet. Schlüssige und umfassende Konzepte für eine Agrarwende fehlen weitgehend. Zugleich stehen die politisch Verantwortlichen, allen voran die neue Verbraucher- und Agrarministerin aber auch der Bundeskanzler, unter hohem Erwartungsdruck. Wohlbedacht scheint Renate Künast aber nicht auf radikale Maßnahmen zu setzen, sondern auf einen behutsamen gleichwohl bestimmten und langfristig angelegten Umbau. Das ist sinnvoll,
Die Agrarwende braucht einen langen Atem

\section{Augen auf und durch}

\section{Wer hätte je geglaubt, dass das Machtkartell aus Agrarlobby, Agrar- politik und Ernährungsindustrie fast über Nacht aufbrechen würde? Die Politik muss nun das entstandene} Zeitfenster entschlossen nutzen. Wichtiger als ein brachiales Umsteuern in Richtung Öko-Landbau sind langfristige Konzepte für ein "Greening" der konventionellen Landwirtschaft und eine konsequente Verzahnung mit der Naturschutzpolitik.

weil die Strukturen auf den Höfen nicht von heute auf morgen verändert werden können: umweltverträglichere Landmaschinen, angepasste Produktionsverfahren oder artgerechte Stallungen können nicht über Nacht aus dem Hut gezaubert werden. Auch die Veredelung und Weiterverarbeitung der landwirtschaftlichen Produkte ist stark auf wenige große Produktionsstätten konzentriert, die Förderung und Entwicklung einer regional angepassteren Ernährungsindustrie wird viel Zeit kosten. Trotz des langfristigen Zieles werden wir daher die Folgen des alten Subventionsirrsinns zunächst weiterhin spüren.

Eine zukunftsfähige Landwirtschaft muss auf mehreren Säulen aufbauen: umweltgerechte Produktion unter Berücksichtigung regionaler Besonderheiten, Qualitätssicherung und -kontrolle, Transparenz und Sicherheit für die Verbraucher sowie Erhaltung der Wettbewerbsfähigkeit.

\section{Schrittweise Ökologisierung}

Wichtigstes Ziel ist dabei die schrittweise Ökologisierung der konventionellen Landbewirtschaftung. Die EU hat mit der Agenda 2000 Spielräume für die Mitgliedsstaaten geschaffen, Subventionen an die Einhaltung bestimmter Umwelt- und Qualitätskriterien zu koppeln. Dies gilt vor allem für den abiotischen Ressourcenschutz, wie etwa eine Begrenzung des Düngemitteleinsatzes. Ins- besondere Deutschland und Frankreich haben diese Möglichkeit bisher nicht genutzt. Hierin liegt auch die Chance, Agrar- und Naturschutzpolitik stärker zusammenzuführen. Enttäuschend ist daher der kürzlich veröffentlichte Entwurf für die Novelle des Bundesnaturschutzgesetzes: Das Haus von Umweltminister Jürgen Trittin hat es versäumt die ,gute fachliche Praxis“, die Mindestnorm für die Umweltbindung der Landwirtschaft, neu und verbindlicher als bisher zu definieren. Hier muss das grüne MinisterInnen-Duo schnell unter neuen Vorzeichen nachbessern! Forschungsbedarf besteht hingegen hinsichtlich handhabbarer Mindestkriterien auch für biotischen Ressourcenschutz, etwa die Gewährleistung der regionstypischen Artenvielfalt.

Auf den ersten Blick einleuchtende Forderungen nach einer flächendeckenden Umstellung auf ökologischen Landbau sind derzeit wenig zielführend. Fehlende Absatzpotenziale können nicht per Dekret geschaffen werden. Vielfach fehlt Wissen über geeignete Strategien für langfristige Veränderungen des Verbraucherverhaltens - auch eine Folge der systematischen Vernachlässigung der Konsumforschung in Deutschland. Ein schneller erster Schritt könnte in der Stärkung des einheitlichen Öko-Prüfzeichens und regionaler Qualitätsmarken liegen. So könnten kurzfristig sichtbare Erfolge organisiert und politisch genutzt werden.

Mittel- und langfristig bedeutet die Wende in der Agrarpolitik keine Abnahme des Subventionsvolumens - auch wenn solche Hoffnungen in der aktuellen Debatte immer wieder laut werden. Aber: Erbringt die Landwirtschaft in Zukunft prüfbar ökologische Leistungen für den Erhalt von Natur und Landschaft als Koppelprodukt einer angepassten landwirtschaftlichen Produktion, steht den Zahlungen anders als heute ein gesellschaftlich nachgefragtes Produkt entgegen. Und sie dürften daher auch konform zu den WTO-Regelungen sein - anders als die Gießkannensubventionen heute.

\section{Die Autorlnnen}

Thomas Korbun ist wissenschaftlicher Geschäftsführer, Alexandra Dehnhardt ist wissenschaftliche Mitarbeiterin des Instituts für ökologische Wirtschaftsforschung. Kontakt: IÖW, Potsdamer Str. 105, 10785 Berlin. Tel. 030/ 884594-0, Fax 030/ 8825439, E-mail:Thomas.Korbun@ioew.de, Alexandra.Dehnhardt@ioew.de 
(c) 20I0 Authors; licensee IÖW and oekom verlag. This is an article distributed under the terms of the Creative Commons Attribution Non-Commercial No Derivates License (http://creativecommons.org/licenses/by-nc-nd/3.o/), which permits unrestricted use, distribution, and reproduction in any medium, provided the original work is properly cited. 